\title{
Physiological Identification of Variation Sources of Transthoracic Electrical Impedance during Breath Holding
}

\author{
Yasunobu Hukushima, M.D.
}

\begin{abstract}
SUMmaRY
Variations of transthoracic electrical impedance and their relations with physiological events within the thorax were studied by using electrodes applying constant sinusoidal current at $55 \mathrm{kHz}$ on bilateral chest walls of anesthetized dogs during breath holding. The level of the impedance lowered during decrease of oxygen and elevated during ether gas evaporation in the lungs with steady pulmonary blood flow. The impedance decreased when concentrated saline was selectively infused into the pulmonary vasculature but not into the left heart and the great vessels. The impedance levels also were related inversely with the change of blood flow and resultant blood volume in the lung when the pulmonary blood flow was decreased as during occlusion of the venous return to the right heart, pulmonary trunk and unilateral pulmonary artery. The impedance levels on the bilateral chest walls varied independently by manipulations mentioned above which was employed in the individual lung of each hemithorax. These results clearly suggest that the current flux applied on the chest wall well penetrates through the chest cage and passes preferentially through the lung parenchyma, the conductivity of which depends on the changes in the amount of electrically conductive or resistive materials associated with physiological events within the lungs.
\end{abstract}

\section{Additional Indexing Words :}

Impedance plethysmograph Bioelectric change Cage factor Current flux path Electrically conductive and non-conductive materials Oxygen absorption Pulmonary blood flow Ether evaporation Saline infusion Differential detection

R ECENT progress of medical electronics has ever covered so many fields $\mathbf{R}$ in cardiovascular and respiratory physiologies with new devices and techniques for clinical and research requirements and also stimulated a great deal of interest in the electrical properties of the thoracic constituents.

One of the resultant developments is the system of impedance plethysmography. This device can make continuous recording of the fluctuation of

From the Third Department of Internal Medicine, Faculty of Medicine, University of Tokyo, Tokyo.

Received for publication October 4, 1969. 
electrical impedance over the chest non-traumatically with bipolar or tetrapolar electrodes, and has been mainly applied to measure cardiac output and to moniter respiratory movement of the thorax as a pneumography.

The essential principle of this method consists in the well known fact that a change in volume or in shape of a tissue or of an organ placed between the electrodes along a circuit is to vary its conductivity for a high frequency current and therefore influences the circuit impedance. If a bioelectric event of this kind accompanies the physiological phenomenon, it can be detected from the same pair of electrodes. ${ }^{1)}$ In other words, if a known physiological phenomenon induces a certain expected bioelectric change between the pair of electrodes, they can be tested for their ability of detecting the phenomenon itself.

Since the first application of this technique for cardiac study was done by Atzler and Lehman, many investigators have struggled with a question, on which part of thorax, heart, systemic vessels, lungs or the cage itself be the essential determinant for the instantaneous level of the transthoracic impedance; more technically to say, how the excitation current flux passes through and distributes in the thorax and what compartment of the thorax as most variable conductive factor really reflects on the impedance variation. ${ }^{2)-5)}$ Several investigators have tried to answer this question in partial success by some modifications of electrical conditions in the subjects merely with mechanical means. ${ }^{3 \gamma, 5}$

This paper reports reasonable responses of the transthoracic impedance to several physiological situations selectively established in the pulmonary circulation and evaluates the validity of informations concerning the current flux path and distribution within the thorax and the clinical applicability of this electronic technique.

\section{METHOD}

Mongrel dogs anesthetized with sodium pentobarbital $(25-30 \mathrm{mg}$. $/ \mathrm{Kg}$.) were kept in supine position with hair trimmed chest cage and heparinized. An intermittent positive pressure respirator operated with pure oxygen was managed to maintain their respiration through a tracheal cannula after elimination of spontaneous respiration with succinyl choline chloride. The peak airway pressure was selected at a range of 5 to $10 \mathrm{~cm}$. water. Cardiac catheters were inserted into the pulmonary artery or femoral vein to record pressures under X-ray fluoroscopy. Catheters with balloon were also introduced from femoral or jugular veins to block the venous return to the right heart or unilateral blood flow of the pulmonary arteries depending on experimental designs.

A block diagram of the system to record impedance variations is shown in Fig. 1. Two sets of tetrapolar electrodes of silver discs or 2 pairs of safety-pin electrodes were attached on bilateral chest walls along with mid-clavicular and mid- 


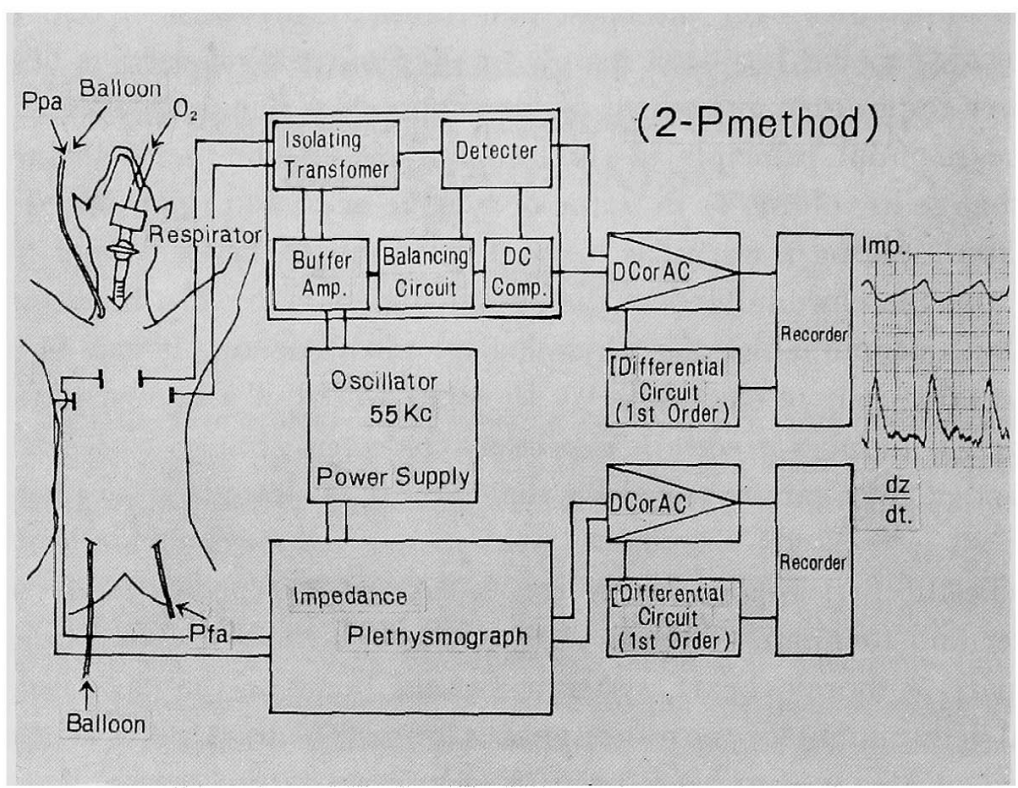

Fig. 1. The block diagram of the impedance plethysmograph. The sinusoidal current of $55 \mathrm{kHz}$ was applied from an oscillator to the individual sides of the chest through 2 pairs of electrodes on the chest walls and double sets of impedance plethysmograph detected potential changes of the hemithoraxes seperately under the constant current. The plethysmograph is composed with an isolating transformer circuit, buffer amplifier, balance control units and DC compensator. Variations of the impedance was resistered with a direct writing pen recorder through a high gain amplifier. See the text for the details.

axillary lines between the 2nd and 4th ribbs both enough away from the cardiac region.

A constant low intensity sinusoidal current at the frequency of $55 \mathrm{kHz}$ from an oscillator was led to the electrodes by an isolating transformer system. Impedance variations were detected as voltage changes between the ipsilateral electrodes on each side of the chest walls through the circuits of phase and amplitude balance control, and DC compensation system of impedance plethysmographs (Modified MPZ-1, Nihon Koden Co., Ltd., Tokyo, JAPAN).

Detected variations of transthoracic impedance were registered continuously through DC amplifiers by a direct writing pen recorder simultaneously with concomitant changes of vascular pressure and ECG record. An increase of impedance was recorded as an upward deflection on the paper. In some cases, the impedance was registered with a tracing of blood flow rate in the pulmonary arterial trunk. A probe of a magnetic flow meter was set around the pulmonary arterial trunk exposed in the opened chest by the median sternal split technique and was balanced in the cage after closure of the chest wall.

The impedance recordings were always performed during breath holding which was carried out by closing the tracheal cannula with a stopcock at relevant levels of ventilation. 
Gains of amplifiers were adjusted so that the maximum range of impedance variations was within $50 \mathrm{~mm}$. on the recording paper.

A total of 315 experiments in 8 thoracotomized open or closed and 17 nonthoracotomized closed chest dogs were carried out in all series of the present report.

\section{RESULTS}

1. Effect of the volume of gas, electrically non-conductive material, in the lung on transthoracic impedance

Impedance changes during breath holding for $2 \mathrm{~min}$. detected with the 2 sets of tetrapolar electrodes on the chest wall are shown in Fig. 2. Levels of the impedance recorded from the right and left side of the chest as depicted on the upper 2 tracings, gradually descended almost alike on straight lines from the moment of breath hold at about the end-tidal level, while EGG in the 3rd showed no change and pressure in the inferior vena cava a minor depression in the bottom. Airway pressures checked by a water manometer also descended seemingly parallelly to the impedance curves as shown in the lower space. These decreases of the impedance level are considered to be due to oxygen decrease, a decrease of electrically resistive component within the thorax, since blood was still perfusing through the pulmonary vessels impinging alveoli filled with pure oxygen and continued to absorb it even during breath holding. A decrease of the airway pressure was due to evacuation of oxygen from alveolar spaces and a delayed retraction of the thoracic wall.

If this change of impedance were due to the retraction of the chest wall,

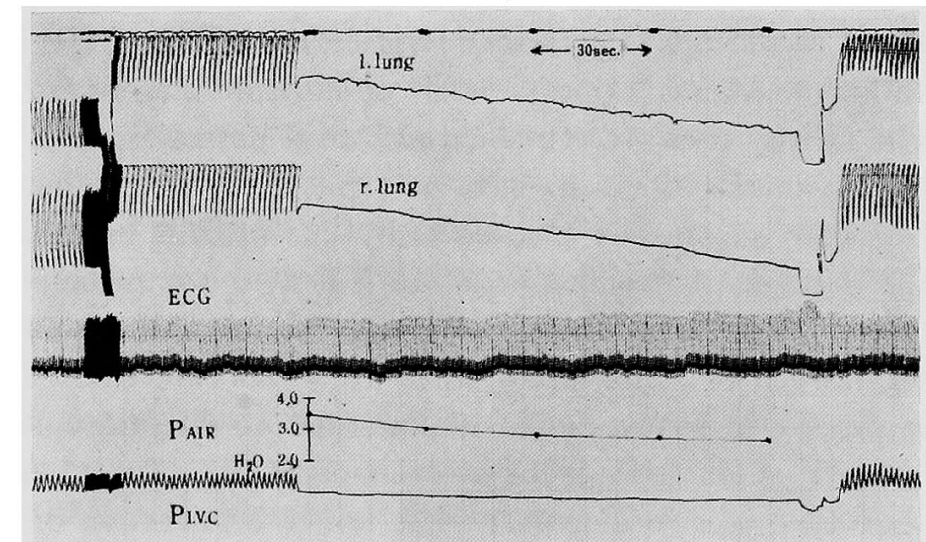

Fig. 2. Impedance curves on the chest walls due to oxygen absorption in the lungs during breath holding for about $2 \mathrm{~min}$. Two impedance curves in the upper space from the right and left lungs, ECG in the middle, airway pressure and the pressure in the inferior vena cava in the lower spaces. Note parallel decreases of the impedance curves and the airway pressure. See the discussion in the text. 


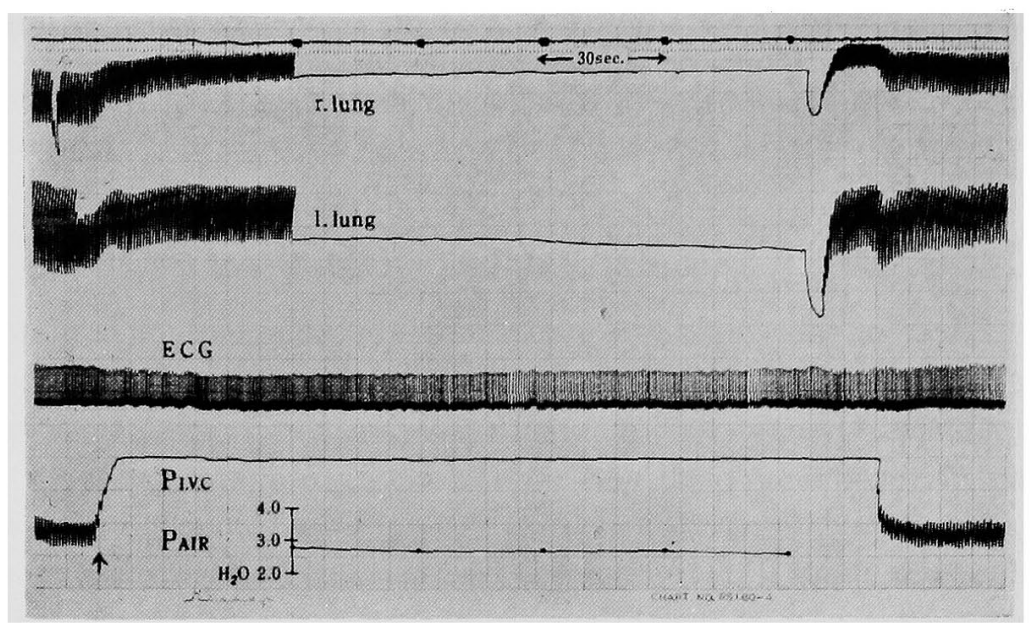

Fig. 3. The impedance curves by oxygen absorption during obstruction of venous return. Note persistence of the levels of impedances and airway pressure on a horizontal plateau because of little absorption due to meagre blood circulation in the lungs.

the parallel decrease of the airway pressure with the impedance should not be expected. From this view point, the descending gradient of impedance curve can be taken to correlate with the ratio of oxygen absorption by the blood in the alveolar space.

This relation was further tested by decreasing the blood volume and flow rate through the lungs (Fig. 3). It was conducted by blocking venous return to the right heart in the superior and inferior venae cavae with a couple of balloons inflated at the tips of catheters. As shown in Fig. 3, the impedance levels in both lungs sustained approximately on horizontal levels during breath holding. The airway pressure remained also in a narrow scope. Elevation of the pressure in the inferior vena cava was due to its obstruction.

This situation was further examined by an infusion method of liquid ether into the pulmonary vascular bed. In Fig. 4, the impedance curves of the upper two made marked bump-like deflection on the way about 5 sec. after the infusion of $2 \mathrm{ml}$. liquid ether from femoral vein. The airway pressure also proceeded in the similar manner as traced in the middle space. The liquid ether evaporates from the alveolar capillaries of the lung and disperses into airway spaces in a moment. A larger evaporation of ether into the alveolar spaces than oxygen absorption from the same space into the blood resulted in increases of gas volume and the pressure within the lung; the former accompanied increase of the impedance which was indicated as an upward deflection on the curve. Again in several sec. the recovery progressed by replacing ether evaporation with oxygen absorption. Around the end seg- 


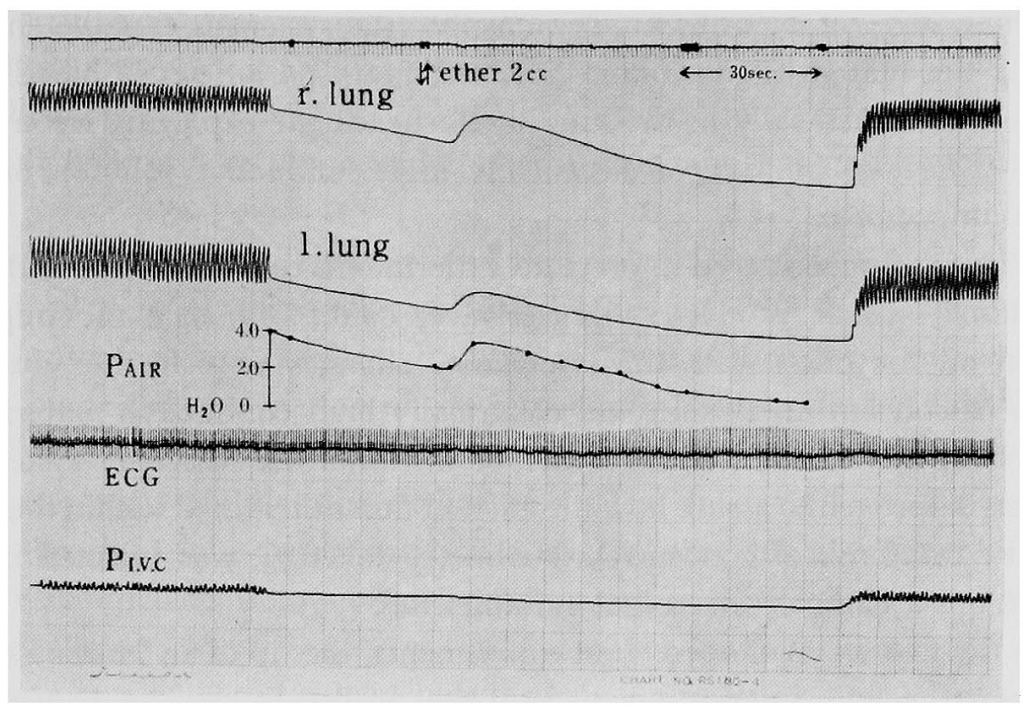

Fig. 4. The effect of liquid ether infusion into the pulmonary arteries during breath holding. See the parallel upward deflection of impedance curves on bilateral chest walls a few sec. after the ether injection. Evaporation of ether, namely accumulation of electrically non-conductive gas in the lungs made the deflections of the curves.

ments of these curves they were appreciably parallel to the extrapolation of the initial segments; namely, the oxygen absorption ratio returned to the original.

It is generally well known that the more blood is in contact with oxygen in vivo, the higher the ratio of oxygen absorption must be. Then, the more blood circulation in the alveolar capillary bed can bring away more oxygen in a period, that is, the oxygen content in an unit volume of the lung structure becomes less and less by absorption in a time. Thus, the proportion of tissue structure which is electrically conductive increases in an unit space, so that the impedance for the current flux through this unit space must become decreased correspondingly. Therefore, the time coursc of gas decrcase within the lung is reflected in a gradient of an impedance decrease, which makes more declining for the higher flow rate of blood. In other terms, the gradient of a descending impedance curve in such conditions must indicate a parameter concerned with blood volume and flow rate in the lungs under the chest electrodes. It is clearly suggested in the result of venous return blocking experiment in which scanty flow of blood through the alveolar vessels would absorb least amount of oxygen and accordingly the impedance stayed through nearly on the same level. The series of ether infusion experiment provided another support for this understanding. 
These types of experiments demonstrate the system is capable enough of detecting the change of gas volume as a secondary parameter of blood volume and flow rate specifically in the lung just beneath the exploring electrodes.

2. Effect of infusion of electrically high conductive solution on transthoracic impedance

Since it was considered in previous experiments that changes of pulmonary gas volume, which is electrically resistive, could influence the impedance recorded on the chest wall, the impedance changes were further studied by infusion of concentrated saline, an electrically high conductive material, into several selected anatomical segments of intrathoracic vascular system as a secondary approach to assure how the current flux distributed within the thorax.

Five to $10 \mathrm{ml}$. of concentrated saline, 5 to $10 \%$, was injected in a few sec. through a catheter located at suitable sites.

In the first series of open chest experiments, the tip of an infusion catheter was located in the inferior vena cava just at the thoracic entrance and 3 sets of bipolar electrodes were fixed directly on the right and left middle lobes of the lung and around the thoracic segment of the inferior vena cava. A forced injection of $5 \mathrm{ml}$. of $10 \%$ saline immediately induced a steep deflection downward on the curve of impedance level on the vena cava and brought out in a few sec. 2 similar changes on the impedance curves from both lung lobes (Fig. 5). These recordings demonstrate reasonable matching of detection

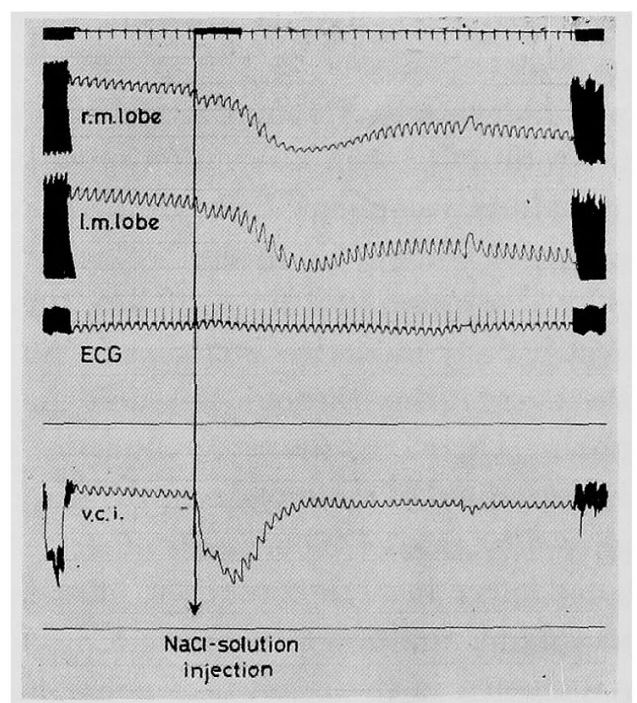

Fig. 5. The impedance changes directly recorded on the lung lobes and the inferior vena cava in open chest experiments after infusion of concentrated saline into the inferior vena cava during breath holding. Electrically high conductive concentrated saline deflected the impedance curves downward. Timing relation of the impedance changes at 3 different sites was reasonably matched in the order. Discussion in the text. 
time at 3 different sites of the leads over the passage of indicator and also even distribution of the saline in both hemithoraxes. The similarity of concave deflection of the upper 2 impedance curves may account for almost equivalent flow rate in the vascular bed of right and left middle lobes of the lung.

The Fig. 6 shows a result of the expcriments, in which blood flow rate was controlled to establish uneven distribution into the lung lobes on each side. Manual occlusion of the extralobar segment of the left pulmonary arterial trunk made slight elevation of the impedance level in the left middle lobe. A following indicator injection drove the impedance curve prominently downward in the right middle lobe exclusively. The enlarged area of this deflection on the right lobe in comparison with the previous experiment (Fig. 5) well corresponded to inflow of large amount of indicator only into the lobes of the right lungs.

These results were further confirmed in the series of closed chest experiments with a pair of bipolar leads on the chest walls (Fig. 7 and 8). All figures show simultaneous recordings of the impedance curves from the left and right lungs and ECG from the top to the bottom. On the left side of Fig. 7, 2 identical concave deflections on the impedance curves were observed by the infusion of $5 \mathrm{ml}$. of $10 \%$ saline into the right ventricle. On the right side of the figure, the same indicator was injected directly into the left pulmonary artery through a catheter and the infusion resulted a distinct unilateral

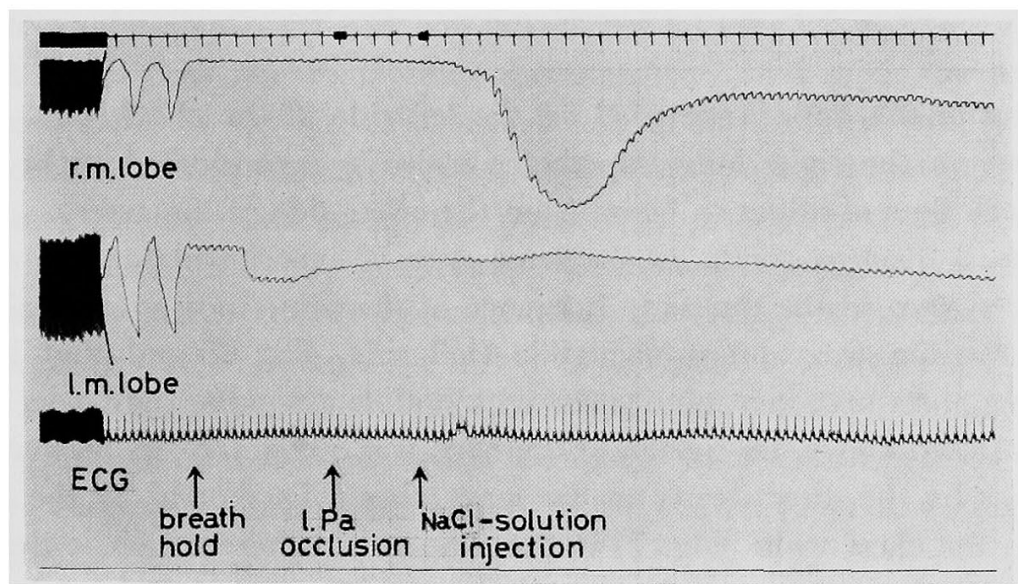

Fig. 6. Change of the impedance recorded directly on the surfaces of bilateral lung lobes after infusion of concentrated saline into the inferior vena cava. Before the infusion, the left main pulmonary artery was occluded manually. A marked downward deflection of the impedance curve was observed solely on the record from the right middle lobe, but not from the left middle lobe in which the perfusion of saline was blocked. See the text for the discussion. 


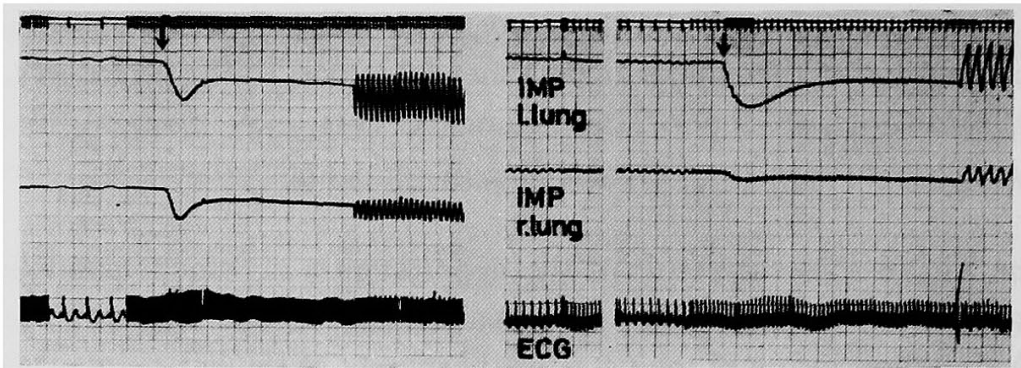

Fig. 7. Selective infusion of concentrated saline into the right ventricle (left side) and into the left main pulmonary artery (right side). Though identical changes of the impedance were observeed on the records from bilateral chest walls in the former experiment, the change was quite prominent solely on the left side of the chest wall in the latter. See the text for further discussion.

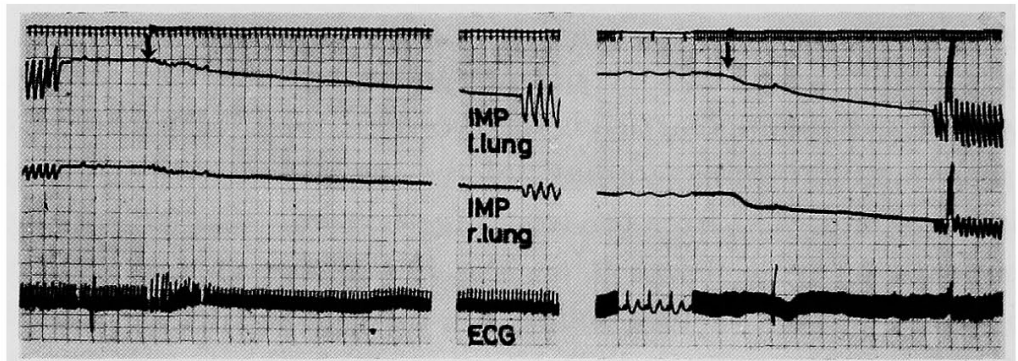

Fig. 8. Selective infusion of concentrated saline into the left atrium (left side) and into the ascending aorta (right side) through a catheter in the closed chest experiments. No definite change of the impedance as observed in Fig. 5, 6 and 7 was recognized in these experiments. Further discussion in the text.

deflection of the impedance level on the left side of the chest wall. A tiny downstep on the right lung impedance curve was considered to be due to small back flow of saline or blood from the other side of the artery.

Fig. 8 displays results of the same type of experiments on the systemic vascular system in the thorax. Influence of the same indicator infusion into the left atrium was almost negligible (left side, Fig. 8) and that into the ascending aorta certainly minor even with double amounts (right side, Fig. 8).

As stated above, no essential difference was recognized in between these detections by the direct leads on the lung lobes (Fig. 6) and by the indirect leads on the chest walls (Fig. 7) in the shape. Furthermore, it is clear from these results that this system does not detect changes of electrical conductivities in the left heart and the great vessels induced by saline infusion (Fig. 8). These results of this series with those in the previous experiments well warrant valid possibility of differential detection of the intrathoracic impedance variations induced with gas or concentrated saline in the lung and not in the left 


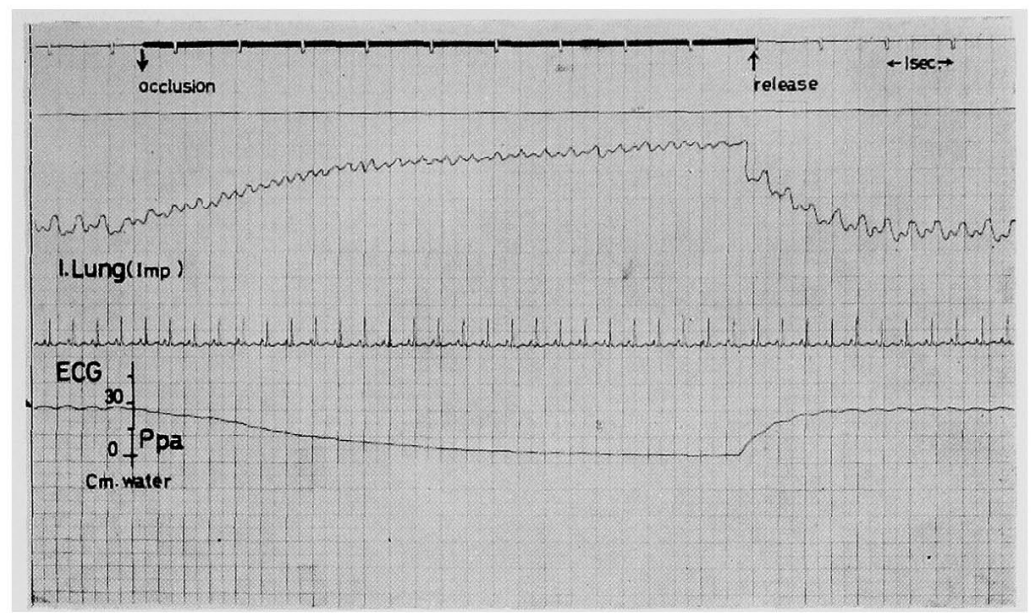

Fig. 9. Impedance change on the left chest wall on occlusion of the left main pulmonary artey in the closed chest experiments. Gradual elevation of the impedance level in the left lung contrasted against the slow fall of the pressure in the left pulmonary artery. Note accompanied flattening of impedance pulse contours during occlusion. See the text for discussion.

heart and great vessels by the regional pairs of electrodes on the chest wall.

3. Effect of blood volume and flow rate variation in the lung on transthoracic impedance

In the following series of experiments, the arrangements were so designed as to modify blood volume and flow rate directly in the lung under detection. A balloon at the tip of a catheter was introduced into the left pulmonary artery to block blood flow and to moniter the pressure. In Fig. 9, the pulsating impedance curve on the left chest wall began to elevate own level gradually up on occlusion with sequent deformation of wave pattern until release of occlusion. On the other hand, the pulmonary arterial pressure descended down to a plateau just close to the zero baseline. Release of occlusion in the left pulmonary artery rebuilt about in 2 sec. the original wave form and the initial pressure level. Because the inflow of the blood was obstructed and the outflow tract was kept open in the obstructed side, it is logically expectable that the content of the blood within the pulmonary vasculature of the left lung was decreased during obstruction. This was reflected in falling of the pressure in the left pulmonary artery.

The impedance changes on the right chest wall during occlusion of the trunk of the left pulmonary artery were lowering of the mean level of impedance pulsation and enlarged pulse contours with higher fluctuation (Fig. 10). These changes disappeared and returned to the original when the obstruction was released. The blood volume and flow rate in the vascular bed in the right 


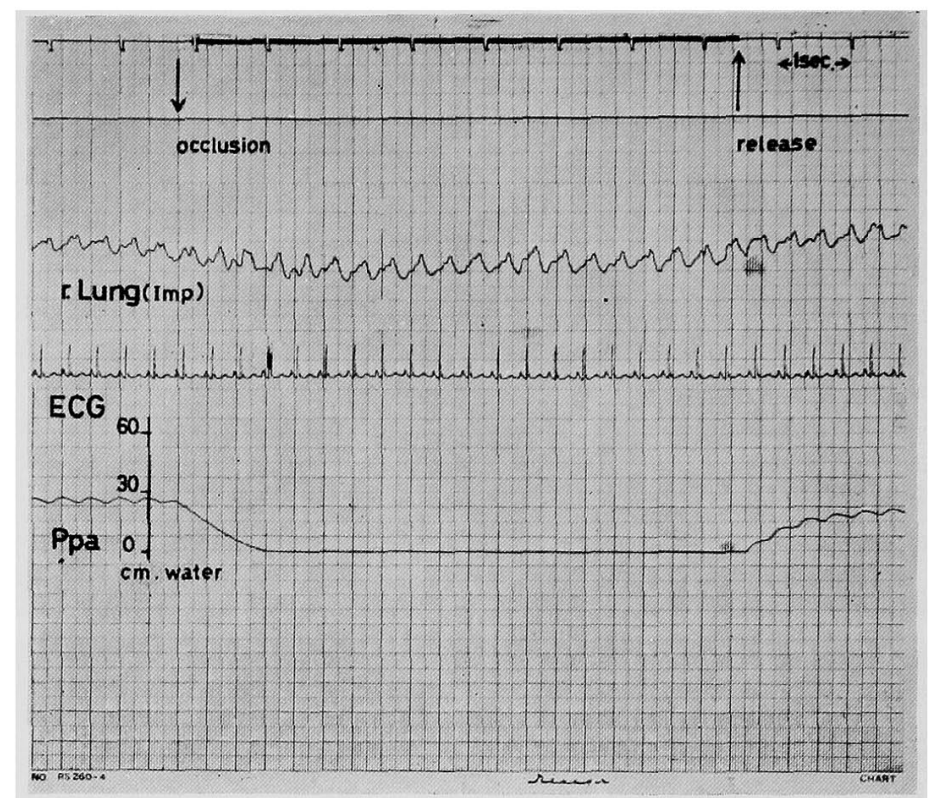

Fig. 10. Impedance change on the right chest wall in the closed chest experiments during occlusion of the left main pulmonary artery. Impedance decrease of the right lungs was accompanied with fall of the pressure in the left pulmonary artery. Prominent gains of impedance pulses were observed simultaneously.

lungs are, of course, expected to be increased through shift of the blood flow from the occluded side.

Another way of blood volume control was carried out by blocking venous return to the right heart. A couple of balloons inflated at the tips of catheters dammed blood return at the superior and inferior entrance of venae cavae into the right atrium. The impedance levels on bilateral chest walls simultaneously run up on the obstruction accompanying decrease of the pulmonary arterial pressure (Fig. 11) and increase of the pressure in the distal portion of obstructed vena cava (Fig. 12). This manipulation definitely diminish the output from the right heart, in other words, the blood inflow into the bilateral pulmonary vasculature, the diminution of which was reflected in falling of the pulmonary arterial pressure and gradual raising of the venous pressure due to a dammed blood in the peripheral portion of the vena cava system. These are the results identical with the impedance changes observed on the left lung during obstruction of the left pulmonary artery (Fig. 9). Obvious emaciation of pulse contour on the impedance curve from the right lung in Fig. 11 is probably due to an effect of a catheter obstructive to the blood flow in the vascular space and also may demonstrate itself the fidelity of chest electrodes for differential detection. 


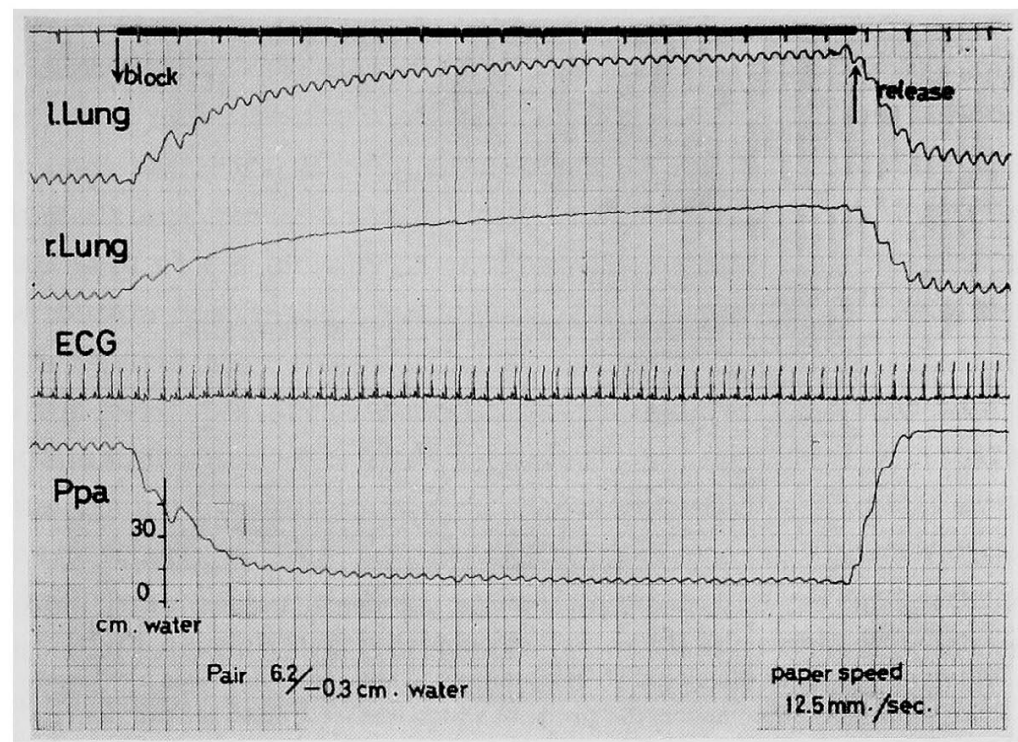

Fig. 11. Impedance elevation on bilateral chest walls during occlusion of venous return in the closed chest experiments. The change of the impedance was associated with fall of the pulmonary arterial pressure. Compare this figure with the next.

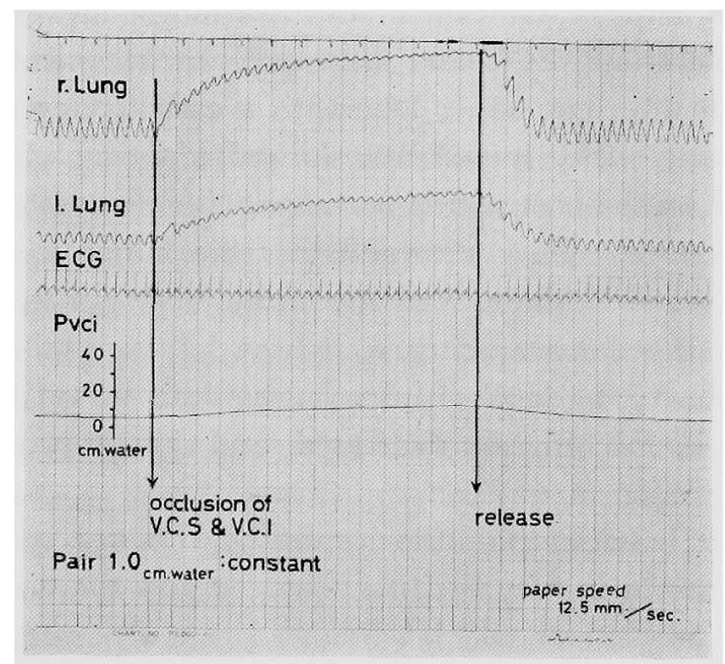

Fig. 12. Impedance elevations and pressure increase in the femoral vein during blocking of venous return in the closed chest experiments. See the details in the text.

Such findings were furthermore examined by installation of a probe of magnetic flow meter around the pulmonary arterial trunk. The chest wall was closed again and set with the bipolar electrodes bilaterally for impedance recording. The result is shown in Fig. 13 from the top to the bottom with 


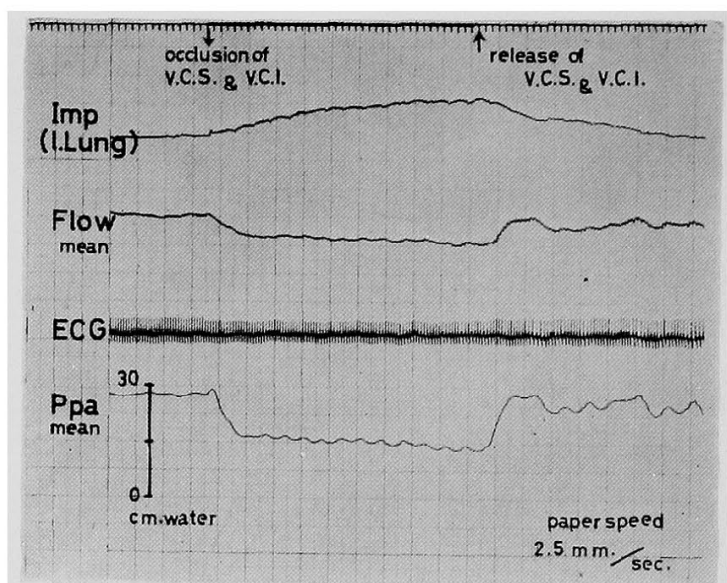

Fig. 13. Simultaneous changes of the impedance, the rate of the pulmonary blood flow and the pulmonary arterial pressure during obstruction of venous return in the closed chest condition. The blood flow was measured with a magnetic flow meter, the probe of which was set around the pulmonary arterial trunk. Elevation of the impedance was synchronized with decreases of the flow and the pulmonary arterial pressure. See the text for further discussion.

recordings of the impedance level in the left lungs, mean flow rate in the pulmonary arterial trunk, ECG and mean pressure measured at a pulmonary arterial branch in the right upper lobe with a catheter inserted from outside during thoracotomy. The impedance showed identical changes with those in Fig. 11 and 12 on the obstruction accompanying decrease of the mean flow rate and the pressure. They returned to their initial levels on release of obstruction. A reversed pattern of the mean flow curve is comparatively analogous to the impedance tracing.

As shown above, the level of impedances shifts upward when the blood flow and volume in the lung are decreased, and shifts downward when those are increased.

The relation between pulsatile impedance change recorded at higher amplification as shown in Fig. 9, 10,11 and 12 and the flow wave recorded with a magnetic flow meter will be discussed in a separate report.

\section{DisGussion}

Transthoracic electrical impedance has been studied by many investigators on the basis of resistivities of tissue components of the chest in vivo for theoretical analysis and raised much arguments on the permeability of high frequency current into the thorax beyond the cage barrier. Thus far, it is 
a majority of opinions that attempts to determine current flux path in a complex geometry such as human thorax does not appear rational at this time. However, they made observations and measurements of transthoracic impedance changes with equivalent techniques and still consider that the genesis of the impedance change must depend ultimately upon physical phenomena associated with physiological events, which induce modification of the current flux distribution between the electrodes, which manifest itself as a measurable change in electrical potential across the electrodes. ${ }^{4)}$

In the present series of experiments, it is tried to identify and evaluate the fundamental cause of transthoracic impedance changes by separating the individual component factors of the accompanying physiological phenomena. Since every recordings of the impedance in the present report were obtained during breath holding, the effects to the impedance of geometrical changes of the chest wall and unstable contact of electrodes over the skin on respiratory movements were eliminated.

In the first series of experiments, the detector explored out that the impedance levels of both hemithoraxes gradually lowered along straight lines seemingly parallel to the airway pressure during breath holding comparatively for long time of $2 \mathrm{~min}$. (Fig. 2). This parallelism between these 2 factors was found steadily in repeated experiments and also in the similar way, when the pulmonary blood flow and volume were reduced by blocking venous return (Fig. 3).

The probable causes of this impedance decrease may be due to (1) geometrical changes of lead distance on shrinking of the cage resulting from decrement of gas volume within the lung, ${ }^{4 \prime}$ (2) increment of conductive factor such as blood in the tissue structurc on the flux path, and (3) relative increase of tissue conductivity on the current path by lessening of lung gas content. If the first possibility be the case, there must be less reduction in the airway pressure presumably by compensation with prompt shrinkage of the thorax. The shrinkage became certainly evident when breath holding was kept longer than 5 min., ${ }^{8}$ but the $2 \mathrm{~min}$. of breath holding is too short to consider change of the circumference of a cage as a main cause of impedance modification, though Geddes and Baker take this factor considerably much in respiratory act.4) Blood volume increase in the lung is not on the point as in the second possibility, because, if so, oxygen absorption could be accelerated progressively, then the curves of impedance level and airway pressure would not run down on straight lines. For all, the third reason seems to be the most problable, and these results could be interpreted as that the oxygen absorption in the alveolar space into the capillary blood produced such linear decrease of the impedance level of both hemithoraxes. Therefore, it is consid- 
ered that the gradients of the curves can be either a parameter for oxygen absorption or secondary indicator for the blood flow rate in the lung.

The other cage factors are the magnitude of skin impedance and contact impedance between skin and electrodes. If they were so great comparatively with changes of impedance induced by physiological events within intrathoracic organs, it should not be possible to obtain any informations implicating with these physiological events by this method.

This point was examined by an indicator infusion procedure with open and closed chest experiments. Results insisted nothing particular other than minor relative lessening of response magnitude in the impedance of hemithorax recorded on the chest wall as observed in Fig. 5,6 and 7. Thus, the cage factor should not be a persistent veil for exploring impedance variations in brief period inside the thorax purposely fixed in an apnic state.

One of the most interesting subjects is the differentiation function of this technique for regional physiological events. Nyboer, 9) Geddes and Baker, , 10, Kinnen, Kubicek and Patterson ${ }^{31}$ and Bonjer ${ }^{51}$ have reported many results of them on different recordings with various combination or arrangements of the electrodes but stated nothing on the fidelity of local electrodes. ${ }^{8}$ Electrodes applied on the chest wall as mentioned in this paper could inform the change of blood conductivity as added with highly concentrated saline and the change of volume and flow rate selectively induced in the individual lung within bilateral hemithoraxes at reasonable timing with notable exactness. They were amazingly indifferent to the state of vessels in the mediastinum; engorgement of venac cavae and concentrated saline infusion in the left atrium, ascending aorta and inferior vena cava (Fig. 5, 6, 7, 8, 9, and 10).

These results demonstrate distinctly the applicability of this technique to obtain differential detection of local information from the individual lung within bilateral hemithoraxes under the electrodes on the chest wall and strongly suggest that the current flux applied from the chest wall passes preferentially through the lung by penetrating the chest wall and support the previous results (Fig. 2, 3 and 4).

The sensitivity of this method in the present experiments depends, of course, on the power of system units, which were so highly qualified as being able to pick up the effect of a catheter on blood flow in a pulmonary artery as well as the ratio of gas exchange in the alveolar space.

Levels of the impedance were elevated on the occluded side of the chest but lowered on the other side during occlusion of the unilateral pulmonary artery (Fig. 9 and 10) and as well elevated simultaneously on bilateral chest walls during obstruction of the venous return (Fig. 11 and 12) promptly on the manipulations even during short hold of breath in a couple of sec., during 
which oxygen absorption could be regarded as being negligible. Since there were no special manipulations applied to manage the outflow tract of pulmonary vascular bed in these experiments, blockades of the inflow into the lung on venae cavae, of the flow in the unilateral pulmonary artery and of the output from the right ventricle on the trunk (Fig. 13) evidently induced decrease of the blood volume in the peripheral pulmonary vascular bed to the obstruction. This situation was verified by decrease of the pulmonary arterial pressure in these experiments. Furthermore, the impedance was decreased on the non-occluded side of the chest wall, on which side the blood flow and volume were increased, because the blood had been moved away from the occluded side." Thus, it can be said that the impedance shifts its level inversely with the change of the blood volume. This statement is quite reasonable and comprehensive, because the blood is a major conductive component within the thorax, ${ }^{11}$ ) so the current can easily pass through the lung when the electrical conductive compartment is expanded in the structure.

\section{Conclusion}

Transthoracic electrical impedance was investigated with physiological procedures such as oxygen absorption, ether and concentrated saline infusion and selective blocking of blood inflow in venae cavae and pulmonary artery in animal experiments. The results confirmed an inversely proportional correlation of transthoracic impedance with the blood volume in the lung vascular bed during breath holding. Therefore, the level of impedance curve can be regarded as a parameter indicative of blood volume in the lung.

The impcdancc technique of such refined qualification as in this report is well expected to have ample function of searching the transthoracic impedance for analytical study. Some technical progress and improvements such as stable quantification of measurement and standardization of simpler electrodes may expand vast and hopeful scope for application both in clinical and research fields in future.

\section{ACKNOWLEDGEMENT}

The author wishes to express his heartful gratitude to Prof. K. Nakao, the director of the Third Department of Internal Medicine and Prof. M. Oshima in the Institute of Medical Electronics and Engineering for their persistent interest and guidance, and also wishes to make many thanks to Dr. S. Kira and his colleagues for the elaborated helps and cooperations in the works.

This work was aided by a grant for Research in Medical Electronics and Biomedical Engineering from the Ministry of Health and Welfare. 


\section{REFERENGES}

1. Geddes, L. A. and Hoff, H. E.: Proceedings of San Diago Symposium for Biomedical Engineering, p. 115, 1963.

2. Kinnen, E., Kubicek, W., Hill, P., and Turton, G.: Technical Documentary Report NO. SAM-TDR-64-5, 1964.

3. Kinnen, E., Kubicek, W., and Patterson, R.: Technical Documentary Report NO. SAMTDR-64-15, 1964.

4. Baker, L. E., Geddes, L. A., Hoff, H. E., and Chaput, G. J.: J. Appl. Physiol. 21: 1491, 1966.

5. Bonjer, F. H., van den Berg, Jr., and Dirken, M. N. J.: Circulation 6: 415, 1952.

6. Witsoe, D. A. and Kinnen, E.: Med. Biol. Engng. 5: 239, 1967.

7. Kira, S., Hukushima, Y., Nakao, K., Kawakami, K., Watanabe, A., and Oshima, M.: Proceedings of the International Union of Physiological Sciences, 1968.

8. Hukushima, Y., Kira, S., Nakao, K., Kawakami, K., Watanabe, A., and Oshima, M.: Iyodenshi-Seitaikogaku Kenkyukai MBE 67-28 (1968-2) (in Japanese).

9. Nyboer, J.: Electrical Impedance Plethysmography, C. C. Thomas Publisher, Illinois, p. 174, 1959.

10. Geddes, L. A. and Baker, L. E.: Med. biol. Engng. 5: 271, 1967. 